\section{Atlas of PET/MR Imaging in Oncology}

O. Ratib, M. Schwaiger, T. Beyer, eds.

New York, NY: Springer, 2013, 232 pages, $\$ 179$

Following the path of clinical applications of hybrid PET/CT, it would seem logical that PET/MR imaging can provide an innovative and attractive alternative taking full advantage of the superiority of MR imaging over CT in differentiating soft-tissue characteristics with a reduction of radiation exposure. The challenges of PET/MR imaging are still numerous both on the technical side and on the clinical side.

Atlas of PET/MR Imaging in Oncology, edited by internationally known experts in the fields of PET and MR imaging, highlights the potential applications of whole-body hybrid PET/MR imaging in oncology. Although it is a convincing collection of anecdotal cases, it reflects only the early observations of 2 academic centers that were first adopting this new technique in clinical practice.

The book is divided into 2 parts, with the first part consisting of 3 chapters that introduce hybrid imaging, PET/MR instrumentation, and technical principles and cover the protocols for standard PET/MR imaging acquisition. The second part has 9 chapters illustrating head and neck, prostate, breast, and gynecologic cancers; brain tumors; pediatric oncology; lymphomas; endocrine tumors; and degenerative and inflammatory diseases. Each case study includes a brief introduction, clinical history, imaging findings, and teaching points, followed by comments. The images are of good quality, and the references are updated. The multimedia version of the book also includes dynamic movies that allow the reader to browse through series of rotating 3-dimensional images, display blending between PET and MR imaging, and visualize 3-dimensional image volumes. The movies can be played either continuously or sequentially for better exploration of sets of images.

The diversity of cases and broad scope of clinical domains covered in the different chapters underline the potential applications not only in oncology but also in other clinical domains. The use of different radiolabeled tracers such as ${ }^{18} \mathrm{~F}$-fluorocholine and ${ }^{18}$ F-fluorotyrosine also shows the potential of PET beyond the conventional ${ }^{18}$ F-FDG tracer in clinical applications of hybrid PET/MR imaging. This new imaging modality has only recently been introduced for clinical use and will face the same challenges and skepticism that PET/CT encountered. The lack of a tangible added value from combined PET/MR imaging over the two examinations acquired separately is the first issue that needs to be addressed from both a clinical perspective and a cost-effectiveness point of view.

This is the first book on PET/MR imaging in oncology. I highly recommend it to trainees and practitioners in the fields of nuclear medicine and radiology, as well as oncology.

E. Edmund Kim
University of California at Irvine
101 The City Dr. S.
Orange, CA 92868
E-mail: edmundek@uci.edu

Published online Aug. 14, 2014. DOI: 10.2967/jnumed.114.145888 